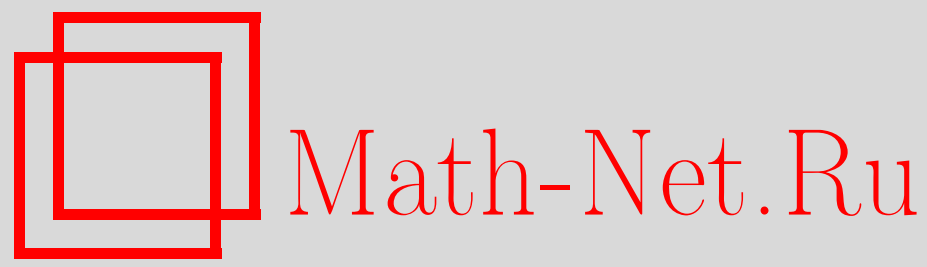

А. М. Вершик, М. И. Граев, Когомологии в неунитарных представлениях полупростых групп Ли (группа $U(2,2))$, Функи. анализ и его прил., 2014, том 48, выпуск $3,1-13$

DOI: https://doi.org/10.4213/faa3144

Использование Общероссийского математического портала MathNet.Ru подразумевает, что вы прочитали и согласны с пользовательским соглашением

http://www.mathnet.ru/rus/agreement

Параметры загрузки:

IP : 54.92 .164 .108

26 апреля 2023 г., 18:02:01

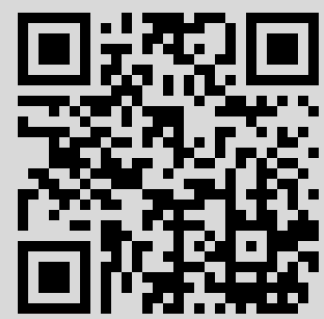


Функциональный анализ и его приложения

2014, т. 48, вып. 3, с. 1-13

УДК 517.5

\title{
Когомологии в неунитарных представлениях полупростых групп Ли (группа $U(2,2))^{*}$
}

\author{
(C) 2014. А. М. ВеРшик, М. И. ГрАЕВ \\ К столетию со дня рождения Израиля Моисеевича Гельфанда
}

\begin{abstract}
Предлагается метод построения особых неунитарных представлений полупростых групп Ли с помощью представлений подгрупп Ивасавы. В качестве характерного примера изучается группа $U(2,2)$.
\end{abstract}

\section{§1. Введение: обзор теории особых представлений}

1.1. Группы токов и особые представления. Группой токов $G^{X}$, где $X$ - топологическое пространство с вероятностной борелевской мерой $m$, а $G-$ произвольная локально компактная группа, называется группа непрерывных отображений $X \rightarrow G$ с поточечным умножением и с тем или иным условием интегрируемости по мере. Изучение представлений групп токов инициировано как самой теорией представлений, так и приложениями к математической физике. Хорошо известная модель неприводимых представлений групп токов $G^{X}$ - фоковская, или гауссовская, в которой существенную роль играют ненулевые 1-когомологии группы коэффициентов (группы $G$ ) со значениями в неприводимых унитарных представлениях этой группы. Такое неприводимое представление $T$ группы $G$ в гильбертовом пространстве $H$ обладает нетривиальным 1 -коциклом, т. е. непрерывным отображением $b: G \rightarrow H$, удовлетворяющим условию

$$
b\left(g_{1} g_{2}\right)=T\left(g_{1}\right) b\left(g_{2}\right)+b\left(g_{1}\right) \quad \text { для любых } g_{1}, g_{2} \in G
$$

и условию нетривиальности: не существует вектора $\xi \in H$, такого, что $b(g)=$ $T(g) \xi-\xi$ для любого $g \in G$. Такие представления называются особыми. Единичное представление является особым для всех групп с нетривиальными комплексными аддитивными характерами, поскольку комплексный аддитивный характер и является нетривиальным 1-коциклом в одномерном единичном комплексном представлении.

В других терминах особое представление характеризуется существованием почти инвариантных векторов; последнее означает, что для всякого $\epsilon>0$ и любого компакта $K$ в группе $G$ существует такой вектор $h$ в пространстве представления, что $\left\|U_{k} h-h\right\|<\epsilon$ для всех $k \in K$; здесь $U_{k}$ - оператор представления, отвечающий элементу $k$.

По теореме [1] всякое особое представление компактно порожденной группы обязано быть не отделимым по Хаусдорфу от единичного представления в топо-

*A. М. В. поддержан грантами РФФИ 14-01-00373 и ОФИ_м 13-01-12422. М. И. Г. поддержан грантом РФФИ 13-01-00190а. 
логии Фелла. Топология Фелла на пространстве унитарных представлений локально компактной группы $G$ задается следующим образом: открытая окрестность представления $\pi$ в гильбертовом пространстве $H$ определяется числом $\epsilon$, конечным набором $h_{1}, \ldots, h_{k}$ элементов пространства $H$ и компактом $K$ группы $G$ и состоит из всех унитарных представлений $\rho$ группы $G$, в пространстве которых найдутся такие элементы $f_{1}, \ldots, f_{k}$, что

$$
\sup _{i=1, \ldots, k ; g \in K}\left\{\left|\left\langle\pi(g) h_{i}, h_{i}\right\rangle-\left\langle\rho(g) f_{i}, f_{i}\right\rangle\right|<\epsilon\right\} .
$$

Обратное утверждение неверно: не всякое представление, не отделимое от единичного, является особым, т. е. имеет нетривиальные первые когомологии.

В работе [2] Шалом доказал гипотезу, высказанную в [1]: если у локально компактной группы единичное представление не изолировано в множестве всех неприводимых унитарных представлений (т.е. группа не обладает свойством Каждана $T$ ), то хотя бы одно особое представление существует. Доказательство основной теоремы в [2] (см. также [3, теорема 3.2.1]) неконструктивно и поэтому не дает прямого способа нахождения особого представления: мы очень мало знаем о том, как выделить особые представления из множества всех представлений, не отделимых от единичного представления (или «приклеенных» к нему).

Принята следующая терминология: корой (core) данного представления $\pi$ называется множество таких представлений $\rho$, что произвольные открытые окрестности представлений $\pi$ и $\rho$ имеют непустое пересечение. В кору регулярного представления аменабельной группы входят все неприводимые представления, и это характеристика аменабельности. Особенно важно использование этого понятия для неприводимых представлений и, в частности, для единичного представления. Если не оговорено противное, то корой группы называется кора ее единичного одномерного представления ${ }^{1)}$.

Ненулевые когомологии со значениями в представлении называются редуцированными [2], если соответствующий коцикл не является пределом тривиальных коциклов. Редуцированные когомологии существуют только в представлениях, лежащих в коре, но не лежащих в подкорке. Для полупростых групп $O(n, 1), U(n, 1)$ нетривиальные когомологии редуцированы. Существуют ли разрешимые группы с этим условием, авторам неизвестно. Наконец, упомянем для полноты еще одно любопытное понятие - группы со свойством Хагерупа ([3], [4]): это группы с неизолированным единичным представлением (т. е. не обладающие свойством Каждана), но у которых коциклы в особом представлении удовлетворяют специальному свойству невырожденности: коцикл как отображение группы в гильбертово пространство

$$
\beta: G \rightarrow H
$$

1) Более детальная терминология: представления, лежащие в коре единичного представления, называются бесконечно малыми (в смысле, аналогичном лейбницевскому; см. [1]), подкоркой данного представления $\pi$ называется множество всех представлений $\rho$, в замыкании которых лежит представление $\pi$ (соответственно подкоркой группы называется подкорка ее единичного представления). В других терминах это означает, что $\rho$ слабо содержит $\pi$ (в частности, единичное представление). Подкорка есть, очевидно, подмножество коры. Интерпретация введенных понятий в терминах хаусдорфовых аксиом отделимости единичного представления и некоторого представления $\rho$ такова: единичное представление лежит в коре представления $\rho$, если не выполнена аксиома отделимости $T_{2}$; для точек подкорки не выполнена даже аксиома отделимости $T_{1}$, но аксиома $T_{0}$ выполнена всегда, так как единичное представление замкнуто. 
является собственным, т. е. прообраз ограниченного множества гильбертова пространства предкомпактен в группе. Этим свойством обладают все аменабельные группы, свободные группы [4], группы $O(n, 1), U(n, 1)$ и др. (см. [5]). Примеры групп, не обладающим ни свойством Каждана, ни свойством Хагерупа, пока изучены недостаточно.

\section{2. Особые представления групп ранга 1 и их разрешимых под-} групп. Из наиболее важных групп Ли - полупростых - только группы $O(n, 1)$ и $U(n, 1)$, имеющие ранг 1 , обладают особыми представлениями. Остальные полупростые группы (включая, как доказал Костант [6], даже группы $S p(n, 1)$ ранга 1) обладают свойством Каждана: их единичное представление изолировано и фоковская модель построения групп токов неприменима. Заметим, что до сих пор теорема Костанта о $S p(n, 1)$ не имеет геометрического доказательства.

Можно сказать, что анализ представлений групп токов для групп $O(n, 1)$ и $U(n, 1)$ достаточно хорошо развит. Работы в этом направлении начались с пионерских работ И. М. Гельфанда и авторов этой статьи, см. [7]-[9]. Общая схема фоковской модели, независимо от конкретной группы, была ранее рассмотрена Араки [14], см. [15], однако примеров полупростых групп до работы [7] не было. В этих работах, а также в работах [10]-[13] были найдены условия неприводимости представлений групп токов и другие их свойства. Принципиальное значение имели идеи работы [9], в которой для групп ранга 1 был предложен метод сведения задачи к разрешимой подгруппе полупростой группы на примере $S L(2, R)$. Развитие этой идеи в работах авторов данной статьи последних 10 лет привело к новым моделям представлений групп токов, эквивалентным фоковской, но построенным по другим (негауссовским) мерам Леви (см. [16], [17]). Это привело к построению интегральной, а затем пуассоновой и квазипуассоновой моделей представлений групп токов ([18], [19]). Возникла также так называемая бесконечномерная мера Лебега ([22]-[24]), являющаяся наиболее точным континуальным аналогом лебеговой меры на конечномерном положительном октанте. Эта мера тесно связана с гамма-процессом Леви и представляет большой самостоятельный интерес.

\section{3. Разрешимые подгруппы полупростых групп и уточнение раз-} ложения Ивасавы. Известно, что для коммутативных и нильпотентных групп особые представления исчерпываются единичным представлением (см. [11], [12]). Но уже для разрешимых групп эта проблема исследована недостаточно. Нас интересует конкретный класс разрешимых групп, являющихся подгруппами групп $O(p, q), U(p, q)$ и, в качестве первого примера, группы $U(2,2)$. Мы работаем с ее разрешимой подгруппой, которую следует называть группой Ивасавъ. Такая подгруппа может быть определена в произвольной связной полупростой вещественной группе Ли (см. [25], где она названа максимальной связной треугольной подгруппой). Разложение Ивасавы для групп состоит в том, что эта подгруппа является «дополнительной» к максимальной компактной подгруппе.

Разрешимая подгруппа (как и всякая аменабельная группа) обладает особым представлением, и поэтому возможна следующая стратегия построения представлений групп токов полупростой группы: сначала найти особое, унитарное или неунитарное, представление этой разрешимой подгруппы и построить представление ее группы токов, а затем пытаться продолжить особое представление на всю полупростую группу и конструировать продолжение представления на ее группу токов. Для групп ранга 1 этот прием, как отмечено выше, впервые 
использован для $S L(2, R)$ в [9] и позднее исследован подробно в цикле работ авторов последних лет ([18], [19]). При переходе от групп ранга 1 к группам большего ранга эта идея построения по-прежнему остается рабочей.

Мы рассматриваем здесь этот вопрос на конкретном примере группы $U(2,2)$, имея в виду и более общую ситуацию, которая будет изложена в другом месте. Мы подробно описываем здесь подгруппу Ивасавы (группа $P$ далее) для этого случая. Первый вопрос - каковы ее особые представления? Но здесь мы сталкиваемся с новой проблемой: для реализации нашего плана необходимо, чтобы это особое представление подгруппы Ивасавы было точным ${ }^{1)}$. Авторам неизвестно, для каких групп это имеет место. Например, для нильпотентных групп точного особого представления не существует. Этот факт представляет интерес уже для группы Гейзенберга, и он эквивалентен одной из форм принципа неопределенности ${ }^{2}$. Для аффинной группы $\mathrm{Aff}(\mathbb{R})$ бесконечномерное представление (квазиэквивалентное регулярному) является точным особым представлением. Но уже для самых простых трехмерных разрешимых групп (в частности, группы $S$, см. далее) вопрос нетривиален.

Проблема. Для каких групп существует точное неприводимое унитарное особое представление и, в частности, для каких разрешимых групп? Более конкретно: когда существует такое представление для группы Ивасавы полупростой вещественной группы Ли?

Таким образом, главная трудность лежит в построении точного особого представления группы Ивасавы, унитарного или нет. Построение неунитарного точного особого представления для группь $U(2,2)$ есть основной результат этой работы, и авторы не сомневаются, что такое построение можно провести для любой вещественной полупростой группы. Продолжение коцикла на всю полупростую группу и построение группы токов становятся возможными, потому что, как показывают результаты упоминавшихся выше работ, имеются модели (например, пуассонова модель - в отличие от фоковской модели) представления групп токов, которые никак не используют унитарность исходного представления. Вопрос о том, можно ли это сделать в унитарном представлении подгруппы Ивасавы, пока остается открытым.

В заключение этого обзора укажем на несколько иной путь, который ближе к первоначальным работам по группам токов и к классическим работам по теории представлений полупростых групп. Он состоит в следующем. Хорошо известно, что особые представления полупростых групп ранга 1 лежат «в хвосте» дополнительных серий, а дополнительные серии есть у любой полупростой группы. Однако для групп ранга, большего 1, унитарные представления «не доходят» до единичного представления; более точно, унитарность теряется при деформации единичного представления. С другой стороны, известно, что в некоторых случаях для этих неунитарных представлений можно указать незнакоопределенную билинейную форму, инвариантную относительно действия группы. Свойства соответствующего пространства с индефинитной метрикой в этом случае плохо изучены, и вопрос об особом представлении, пусть и неунитарном, по-видимому,

1) Точное (или невырожденное) представление - это представление, ядро которого тривиально.

${ }^{2)}$ Беседы первого автора с В. П. Хавиным на эту тему показали, что упомянутый факт о группе Гейзенберга, как будто, не имеет пока чисто аналитического (не представленческого) доказательства. 
ранее не ставился. Наш альтернативный план состоит в аналитическом продолжении унитарных представлений подгруппы Ивасавы и, в частности, в построении неунитарного особого представления на этой основе. Возможно, что указанные два подхода к теории представлений групп токов для полупростых групп ранга, большего 1, приведут к различным классам представлений.

\section{§2. Группа $U(2,2)$ и ее подгруппа Ивасавы}

2.1. Описание группы $\boldsymbol{U}(\mathbf{2}, 2)$. В качестве первоначального примера мы рассматриваем группу $U(2,2)$ линейных преобразований пространства $\mathbb{C}^{4}$, сохраняющих фиксированную эрмитову форму с сигнатурой $(2,2)$; здесь выбрана эрмитова форма вида

$$
x_{1} \bar{x}_{3}+\bar{x}_{1} x_{3}+x_{2} \bar{x}_{4}+\bar{x}_{2} x_{4} .
$$

Группа $U(2,2)$ является одним из самых простых примеров полупростой группы Ли, вещественный ранг которой больше единицы (равен двум). Группы вида $U(p, q)$ называют псевдоунитарными. В настоящем параграфе мы уточняем разложения Ивасавы для группы $U(2,2)$ и изучаем структуру главного объекта разрешимой подгруппы Ивасавы $P$.

Мы будем представлять элементы $g$ группы $U(2,2)$ в форме блочных 2 × 2-матриц с блоками - матрицами второго порядка,

$$
g=\left(\begin{array}{ll}
g_{11} & g_{12} \\
g_{21} & g_{22}
\end{array}\right)
$$

(где $g_{i j}$ - комплексные матрицы порядка 2), удовлетворяющих соотношению

$$
g \sigma g^{*}=\sigma, \quad \text { где } \sigma=\left(\begin{array}{cc}
0 & e_{2} \\
e_{2} & 0
\end{array}\right) \text {. }
$$

Здесь $e_{2}$ - единичная 2 × 2-матрица, а символ * обозначает в комплексном случае матричное транспонирование совместно с сопряжением, а в вещественном - только транспонирование.

Эти соотношения эквивалентны следующим соотношениям между блоками матрицы $g$ :

$$
g_{12} g_{21}^{*}+g_{11} g_{22}^{*}=e_{2}, \quad g_{11} g_{12}^{*}+g_{12} g_{11}^{*}=0, \quad g_{22} g_{21}^{*}+g_{21} g_{22}^{*}=0 .
$$

Вещественная размерность группы $U(2,2)$ равна 16. Главную роль в дальнейшем будет играть разрешимая подгруппа $P$ группь $U(2,2)$, порожсенная следующими двумя подгруппами $N$ и $S$ :

(1) аддитивной (коммутативной) группой $N$ косоэрмитовых блочных матриц вида

$$
\left(\begin{array}{cc}
e_{2} & 0 \\
n & e_{2}
\end{array}\right)
$$

где $n$ - косоэрмитова матрица второго порядка: $n+n^{*}=0$, и

(2) разрешимой подгруппой $S$ ранга разрешимости 2 блочных матриц вида

$$
\left(\begin{array}{cc}
s^{*-1} & 0 \\
0 & s
\end{array}\right)
$$


где $s$ - нижнетреугольная комплексная матрица с положительными элементами на диагонали:

$$
\left(\begin{array}{cc}
r_{1} & 0 \\
r & r_{2}
\end{array}\right), \quad r_{1}, r_{2}>0, r \in \mathbb{C} .
$$

В дальнейшем мы иногда будем писать $s \in S$, имея в виду, что группе $S$ принадлежит матрица вида (1).

Группы $S$ и $N$ имеют вещественную размерность 4 , а группа $P$ - вещественную размерность 8 .

Общий вид элемента группы $P$ таков:

$$
\left(\begin{array}{cc}
s^{*-1} & 0 \\
X & s
\end{array}\right)
$$

(мы будем иногда записывать его как пару $(s, X))$, где $X$ - матрица второго порядка, удовлетворяющая условию, которое можно было назвать относительной косоэрмитовостъю (относительно матрицы $s$ ):

$$
s X^{*}+X s^{*}=0 \text {. }
$$

Непосредственно проверяется следующее утверждение:

Предложение 1. Группа $U(2,2)$ алгебраически порождена элементами группь $P$ и инволюиией $\sigma$; группы $N$ u пересекаются только по единичному элементу.

Однородное пространство $U(2,2) / K$, где $K$ - максимальная компактная подгруппа группы $U(2,2)$, и есть в точности пространство подгруппы $P$, чем и оправдано название «подгруппа Ивасавы». (Это дает в дальнейшем возможность продолжения коцикла с подгруппы $P$ на всю группу $U(2,2)$.

Поскольку группа $S$, очевидно, действует на аддитивной группе $N$ косоэрмитовых матриц по правилу

$$
n \mapsto \text { sns }^{*}, \quad s \in S, n \in N,
$$

можно определить групповое полупрямое произведение $Q=S \curlywedge N$ групп $S$ и $N$. Непосредственно проверяется следующее важное утверждение:

Теорема 1. Группь $P$ и $Q$ канонически изоморфны; изоморфизм $I: P \rightarrow Q$ задается формулой

$$
I:(s, X) \rightarrow\left(s, X s^{*}\right),
$$

где левая часть есть элемент подгруппь $P \subset U(2,2)$, а правая - элемент полупрямого произведения $Q$ (матрица $X s^{*}$, очевидно, косоэрмитова). Обратный изоморфизм таков: $(s, n) \rightarrow\left(s, n s^{*-1}\right)$, где $n$ косоэрмитова.

Доказательство. Проверка гомоморфности умножения:

$$
\begin{aligned}
I\left(\left(s_{1}, X_{1}\right) \circ\left(s_{2}, X_{2}\right)\right) & =I\left(s_{1} s_{2}, X_{1} s_{2}^{*-1}+s_{1} X_{2}\right) \\
& =\left(s_{1} s_{2}, X_{1} s_{1}^{*}+s_{1} X_{2} s_{2}^{*} s_{1}^{*}\right)=I\left(s_{1}, X_{1}\right) I\left(s_{2}, X_{2}\right) .
\end{aligned}
$$

Отображение $I$ переводит группу $P$ в группу, матричная реализация которой состоит из совокупности пар $(s, X)$, удовлетворяющих соотношению $(2)$. Поэтому изоморфизм полупрямого произведения подгрупп $S$ и $N$, т. е. группы $Q$, с его матричной реализацией, т. е. с группой $P$, не есть прямое произведение тождественного изоморфизма подгруппы $S$ и тождественного изоморфизма 
нормального делителя $N$. Тем не менее группа $P$ есть полупрямое произведение $S<N$ групп $S$ и $N .1)$

Общий вид блока элементов коммутанта группы $S$ в обозначениях, принятых выше, таков:

$$
\left(\begin{array}{ll}
1 & 0 \\
r & 1
\end{array}\right)
$$

где $r$ - комплексное число. Ранг разрешимости группы $S$ равен 2. Таким образом, коммутант группы $P$ есть полупрямое произведение группы $\mathbb{C}$ и коммутативной группы косоэрмитовых матриц (с нетривиальным действием $\mathbb{C}$ ).

Следствие 1. Ранг разрешимости группь $P$ равен трем.

\section{2. Представления подгруппы Ивасавы и почти инвариантные ме-} ры. Наша цель состоит в изучении особого представления подгруппы $P$ и последующего продолжения его на всю группу $U(2,2)$. Мы будем изучать представления группы $P$ как полупрямого произведения.

Группа $N$ изоморфна группе всех своих непрерывных характеров $\widehat{N}$, а группа $S$ действует сопряженными автоморфизмами на группе характеров $\widehat{N} ;$ при этом прямое и сопряженное действия на группе $N$ (если ее отождествить с ее сопряженной) совпадают:

$$
n \rightarrow s n s^{*}, \quad \chi \rightarrow \chi_{s}, \quad \text { где } \chi_{s}(n)=\chi\left(s n s^{*}\right) .
$$

Предложение 2. Имеется четыре орбиты положсительной меры действия группы $S$ автоморфизмами на группе $\widehat{N}$ ( и на группе $N)$ : они параметризуются знаками мнимой части диагональных элементов, т.е. являются соответственно орбитами элементов

$$
\left(\begin{array}{cc} 
\pm i & 0 \\
0 & \pm i
\end{array}\right)
$$

На каждой орбите действие группь $S$ является свободным и точным; поэтому каждую из орбит можно отождествить с группой $S$, а действие совпадает с действием правыми сдвигами группь на себе.

Заметим, что имеются также орбиты меньшей размерности, имеющие меру нуль, но они нам не понадобятся.

Очевидно, что все четыре действия группы $S$ на невырожденных орбитах топологически изоморфны, а соответствующие представления полупрямого произведения отличаются лишь коциклом со значениями в $\mathbb{Z}_{2}$, на который умножаются мультипликаторы; поэтому достаточно рассмотреть лишь одну (любую) орбиту.

Унитарное представления полупрямого произведения группы на коммутативную группу (у нас $P=S \nless N$ ) канонически реализуется следующим образом.

1) В случае группы ранга 1 матричная реализация подгруппы Ивасавы несколько проще; например, для группы $S L(2)$ подгруппа Ивасавы $P$ есть подгруппа треугольных матриц

$$
\left(\begin{array}{cc}
s^{-1} & 0 \\
n & s
\end{array}\right), \quad s \in \mathbb{R}_{+}, n \in \mathbb{R},
$$

и структура полупрямого произведения согласована с обычным матричным представлением, поскольку группа как множество есть прямое произведение нормального делителя, состоящего из матриц вида $\left(\begin{array}{ll}1 & 0 \\ n & 1\end{array}\right)$, и подгруппы матриц вида $\left(\begin{array}{cc}s^{-1} & 0 \\ 0 & s\end{array}\right)$. 
Рассмотрим вероятностную меру $\mu$ на группе характеров (т. е. на группе $\widehat{N}$ ), квазиинвариантную относительно действия (группы $S$ ). Все такие меры эквивалентны, поскольку группа $S$ локально компактна и действует транзитивно. Поэтому в гильбертовом пространстве $L_{\mu}^{2}(\widehat{N})$ можно определить унитарные представления группы $P$, индуцированные действием $S$, и представление группы $N$ мультипликаторами, т. е. операторами умножения (на характеры группы $\widehat{N}$, совпадающей с исходной группой $N$ ). Общие представления реализуются в векторнозначном пространстве $L_{\mu}^{2}(\widehat{N})$, но мы их не рассматриваем.

Неприводимость приведенного представления полупрямого произведения равносильна эргодичности меры $\mu$, и в силу сказанного выше можно было бы считать, что орбита есть сама группа $S$, т. е. рассматривать представление группы $S$ в пространстве $L_{\mu}^{2}(S)$ по некоторой квазиинвариантной мере $\mu$ относительно правого действия группы.

Такие представления принадлежат коре группы $P$ - это следует из того, что они в совокупности квазиэквивалентны регулярному представлению группы $P$, которое (в силу аменабельности группы $S$ ) слабо содержит единичное представление. Кроме того, коре принадлежит единичное представление, а также особые представления группы $S$, которые можно рассматривать как представления группы $P$, поскольку $S=P / N$. Исчерпывается ли кора этим списком, неизвестно, как неизвестно, являются ли построенные четыре представления особыми для группы $P$, иначе говоря, есть ли в них почти инвариантный вектор. Но сначала выясним структуру особых представлений группы $S$.

Лемма 1. Группа $S$ имеет континуум особых унитарных представлений, параметризуемых точками из $\mathbb{C}$, лежащими на единичной окружности (характерами). Точных особых представлений у группы $S$ нет.

Доказательство. Аффинная группа $\mathrm{Aff}(\mathbb{R})$, т. е. группа матриц

$$
\left(\begin{array}{cc}
e^{a} & 0 \\
b & e^{-a}
\end{array}\right), \quad \text { где } a, b \in \mathbb{R},
$$

является группой Ивасавы для $S L(2, R)$ и играет для нее ту же роль, что и группа $P$ для группы $U(2,2)$. Заметим, что как подгруппа группы $S L(2, R)$ она есть полупрямое произведение $\mathbb{R}_{+}$и $\mathbb{R}$, согласованное с матричным представлением. Как сказано выше, у нас это не так. Особые унитарные точные представления группы Aff $(\mathbb{R})$ существуют (их два) и продолжаются до особого представления группы $S L(2, R)$.

Рассмотрим группу матриц

$$
\left(\begin{array}{cc}
r_{1} & 0 \\
r & r_{2}
\end{array}\right), \quad r \in \mathbb{C}, r_{1}>0, r_{2}>0 .
$$

Заметим, что если фиксировать детерминант матрицы $\left(=r_{1} \cdot r_{2}\right)$ и характер на группе $\mathbb{C}=\mathbb{R}^{2}$, лежащий на окружности, то полученная подгруппа изоморфно отображается на группу $\mathrm{Aff}(\mathbb{R})$ и поэтому все особые представления группы $\operatorname{Aff}(\mathbb{R})$ поднимаются до особых представлений группы $S$; ни одно из них не является точным; других особых представлений у этой группы нет.

Однако нас интересует не столько группа $S$, сколько группа $P$. Гипотетически, особые унитарные представления группы $P$ можно построить, используя особые (неточные) представления группы $S$ вместе с представлениями группы 
$P$, построенными выше. Пока вопрос об их существовании открыт. Для полноты картины опишем модель гильбертова пространства особого унитарного представления для группы $S L(2, R)$ и ее треугольной подгруппы $P$. Рассмотрим пространство $L_{m}^{2}\left(\mathbb{R}_{+}\right)$( $m$ - мера Лебега на полупрямой). Удобнее перейти к преобразованию Фурье, и тогда нужное представления треугольной подгруппы (записанной в виде группы преобразований $x \mapsto e^{\beta} x+a, \beta, a \in \mathbb{R}$ ) реализуется в $L_{\widehat{m}}^{2}(\mathbb{R})(\widehat{m}$ - лебегова мера на прямой) следующим образом:

$$
\left(U_{a, \beta} F\right)(z)=\exp \left\{i a e^{z}\right\} F(z+\beta), \quad z \in \mathbb{R} .
$$

Почти инвариантный вектор в этой модели есть произвольная функция $f$, удовлетворяющая для любых заданных $t, a, b \in \mathbb{R}$ условиям

$$
\begin{gathered}
f(x)=0, \quad \text { если } x>t \in \mathbb{R} ; \quad f \notin L_{\widehat{m}}^{2}(\mathbb{R}) ; \\
\left(1-\exp \left\{i e^{z} b\right\}\right) f \in L_{\widehat{m}}^{2}(\mathbb{R}) ; \quad f(\cdot)-f(\cdot+a) \in L_{\widehat{m}}^{2}(\mathbb{R}) .
\end{gathered}
$$

Другое более распространенное описание особого представления (см. [7]) в пространстве аналитических функций связано с пределом представлений дополнительной серии при стремлении к единичному представлению.

2.3. Почти инвариантные меры и неунитарные представления. Назовем меру $\nu$ на группе $S$ почти инвариантной (справа), если она бесконечна, абсолютно непрерывна относительно правой меры Хаара на группе $S$ (и, тем самым, квазиинвариантна относительно правых сдвигов $\left.s \mapsto s s_{0}\right)$ и все ее производные $d \nu\left(s s_{0}\right) / d \nu(s)$ определены и ограничены для любого $s_{0} \in S$. (В силу указанного изоморфизма $S \rightarrow H$, где $H$ - произвольная невырожденная $S$-орбита на группе характеров $\widehat{N}$, это определение переносится на меры, сосредоточенные на любой из невырожденных орбит группы $\widehat{N}$ ).

Условию почти инвариантности удовлетворяет, конечно, и мера Лебега на $S$, т. е. $d s=d s_{11} d s_{22} d s_{21} d \bar{s}_{21}$, поскольку $d\left(s s_{0}\right)=\pi(s) d s$, где $\pi(s)=s_{11}^{3} s_{22}$. Отсюда следует, что этому условию удовлетворяет любая мера вида $d \nu(s)=$ $u(s) d s$, где $u(s)$ - произвольная функция, такая, что отношение $u\left(s s_{0}\right) / u(s)-$ ограниченная функция при любом $s_{0} \in S$. В частности, ему удовлетворяет мера $\mu$, инвариантная относительно правых сдвигов (мера Хаара):

$$
d \mu(s)=\pi^{-1}(s) d s, \quad \pi(s)=s_{11}^{3} s_{22} .
$$

Однако нам будет удобнее рассмотреть иную меру.

Пусть на пространстве $X$ действует группа $G$ и на $X$ заданы две эквивалентные квазиинвариантные относительно действия группы $G$ меры $\mu$ и $\nu$. Предположим, что плотность одной меры по другой отделена от бесконечности и от нуля. Рассмотрим в пространствах $L_{\mu}^{2}(X)$ и $L_{\nu}^{2}(X)$ представления группы $G$, порожденные подстановками переменной $\left(U_{g} f\right)(\cdot)=f(g \cdot)$, и представления группы мультипликаторов, по модулю равных единице. Таким образом, мы получаем представления скрещенного произведения группы мультипликаторов и группы $G$. Хорошо известная изометрия между пространствами $L_{\mu}^{2}(X)$ и $L_{\nu}^{2}(X)$, состоящая в умножении функции на квадратный корень из плотности одной меры по другой, коммутирует с мультипликаторами, но не коммутирует, вообще говоря, с действием группы. Эта изометрия широко используется для того, чтобы подправить действие; например, если одна из мер инвариантна 
и, тем самым, определяет унитарное представление упомянутого скрещенного произведения, то и подправленное действие тоже становится унитарным.

Пусть $\nu$ - почти инвариантная мера на $H$. Рассмотрим неунитарное представление группы $P$ в гильбертовом пространстве $L_{\nu}^{2}(S)$, т. е. пространстве функций $F$ на $S$ с нормой $\|F\|^{2}=\int_{S}|F(s)|^{2} d \nu(s)<\infty$. По определению операторы представления подгрупп $N$ и $S$ задаются следующими формулами:

$$
\begin{aligned}
(T(n) F)(s) & =\chi_{k}(n, s) F(s) & \text { при } & n \in N, \\
\left(T\left(s_{0}\right) F\right)(s) & =F\left(s s_{0}\right) & & \text { при } s_{0} \in S .
\end{aligned}
$$

Здесь $\chi_{k}(n, s)$ есть образ характера $\chi(\cdot) \in \widehat{N}$ как функции на $S$ при (единственном) изоморфизме $S$-орбиты в $\widehat{N}$ с номером $k=1,2,3,4$ и группы $S$, сохраняющем действие $S$; различие между четырьмя орбитами сводится к умножению образа на $\pm i$ по каждой из переменных. Из определения следует, что операторы $T(n)$ унитарны, а операторы $T\left(s_{0}\right)$ ограничены в силу почти инвариантности меры $\nu$. Можно переписать формулы более компактно:

$$
\begin{aligned}
(T(n) f)(s) & =\chi\left(s n s^{*}\right) f(s) & \text { при } & n \in N, \\
\left(T\left(s_{0}\right) f\right)(s) & =f\left(s s_{0}\right) & & \text { при } s_{0} \in S,
\end{aligned}
$$

где $\chi$ - некоторый фиксированный характер $S$-орбиты $H$ на группе характеров $\widehat{N}$.

Нетрудно убедиться, что операторы представления подгрупп $N$ и $S$ порождают в совокупности представление всей группы $P$ в пространстве $L_{\nu}^{2}(S)$. В частности, если $\nu=\mu$ - мера Хаара на $S$, это представление унитарно. Обозначим эти представления группы $P$ через $\pi_{k}, k=1,2,3,4 ;$ мы опускаем индекс $k$, поскольку все четыре представления в существенном различаются между собой лишь выбором характера на орбитах.

Теорема 2. Неунитарные представления $\pi$ группы $P$, определенные выше, операторно и пространственно неприводимы. При этом представления, отвечающие разным мерам и разным индексам $k$ пространственно (т.е. унитарно) эквивалентны тогда и толъко тогда, когда меры отличаются множителем $\left(\nu^{\prime}=c \nu\right)$, а индексъ $k$ совпадают.

\section{4. Точное неунитарное особое неприводимое представления груп-} пы Ивасавы. Изучим вопрос о том, как зависят когомологии группы со значениями в пространстве $L_{\nu}^{2}(X)$ от почти инвариантной меры $\nu$. Подчеркнем, что замена меры на эквивалентную и, в частности, унитаризация представлений (см. выше) не индуцируют изоморфизм групп когомологий $H^{1}\left(G, \pi_{\mu}\right)$ и $H^{1}\left(G, \pi_{\nu}\right)$, где $\pi_{\mu}, \pi_{\nu}$ - представления, соответствующие мерам $\mu$ и $\nu$, так как, вообе говоря, пространственная изометрия не переводит коцикл группы со значениями в одном пространстве в коцикл со значениями в другом. Иначе говоря, когомологии групп со значениями в гильбертовых пространствах $L_{\mu}^{2}(X)$ с различными почти инвариантными мерами $\mu$ при данном действии группы, вообще говоря, различны. Этим объясняется наш выбор почти инвриантной меры далее. 
Зафиксируем почти инвариантную меру $\nu$ на пространстве $S$ и введем пространство $Z_{\nu}$ измеримых функций на $S$, удовлетворяющих двум условиям:

$$
\begin{gathered}
\int_{S}\left|f\left(s s_{0}\right)-f(s)\right|^{2} d \nu(s)<\infty \quad \text { для любого } s_{0} \in S, \\
\int_{S}\left|\left(\chi\left(s n s^{*}\right)-1\right) f(s)\right|^{2} d \nu(s)<\infty \quad \text { для любого } n \in N .
\end{gathered}
$$

Очевидно, что $L_{\nu}^{2}(S) \subset Z_{\nu}$.

Элементы $f \in Z_{\nu}$ будут играть роль кограниц, а пространство функций вида $b(g)=T(g) f-f$ есть пространство коциклов. Если $f \in L_{\nu}^{2}(S)$, то $b(g)=T(g) f-f$ есть коцикл, когомологичный нулю. Отсюда следует

Лемма 2. Представление группы $P$ в пространстве $L_{\nu}^{2}(S)$ является особъм тогда и только тогда, когда

$$
L_{\nu}^{2}(S) \varsubsetneqq Z_{\nu}
$$

m. е. когда существует кограница, не лежсащая в $L_{\nu}^{2}(S)$. Таким образом, группа когомологий имеет вид

$$
H^{1}\left(P ; L_{\nu}^{2}(S)=Z_{\nu} / Z_{\nu} L_{\nu}^{2}(S) .\right.
$$

Мера $\nu$ называется особой, если $H^{1}\left(P ; L_{\nu}^{2}(S)\right) \neq 0$, т. е. существуют кограницы, не лежащие в $L_{\nu}^{2}(S)$. Авторам неизвестно, является ли мера Хаара $m$ на $S$ особой и, тем самым, неизвестно, является ли особым естественное унитарное представление группы $P$ на $L_{m}^{2}(S)$. Поэтому мы выбираем другую почти инвариантную меру для построения особого, но уже неунитарного представления группы $P$.

В дальнейшем фиксируется почти инвариантная мера $\nu$ следующего вида:

$$
d \nu(s)=|s|^{-4} d s, \quad \text { где }|s|^{2}=\operatorname{tr}\left(s^{*} s\right)=s_{11}^{2}+s_{22}^{2}+\left|s_{21}\right|^{2} .
$$

Удобно представлять эту меру в полярных координатах на $S$. Для этого заметим, что многообразие элементов $\omega \in S$ с нормой $|\omega|=1$ эквивалентно области на единичной сфере в $\mathbb{R}^{4}$. Назовем сферическими координатами матрицы $s$ из формулы (3) число $r=|s|$ и матрицу $\omega=|s|^{-1} s$. Тогда $s=r \omega$ и выражение меры $\nu$ в полярных координатах имеет вид

$$
d \nu(s)=r^{-1} d r d \omega,
$$

где $d \omega-$ инвариантная мера на сфере.

Непосредственно проверяется следующее утверждение:

Теорема 3. Представление $\pi$ группь $P$ в гилъбертовом пространстве $L_{\nu}^{2}(S)$, где $d \nu(s)=|s|^{-4} d s$, является особым и обладает нетривиальным коииклом вида

$$
b(g)=T(g) f-f, \quad \text { əде } f(s)=e^{-|s| / 2} .
$$

2.5. Продолжение особого неунитарного представления подгруппы $\boldsymbol{P}$ на всю группу $\boldsymbol{U}(\mathbf{2}, \mathbf{2})$. Остается проверить, что особое представление продолжается до неунитарного представления всей группы $U(2,2)$. Конструкция искомого продолжения основана на следующем свойстве группы $U(2,2)$. Любой элемент $g \in U(2,2)$ представим, и притом однозначно, в виде произведения $g=p k, p \in P, k \in K$, где $K$ - максимальная компактная подгруппа элементов 
$k \in U(2,2)$, удовлетворяющих соотношению $k k^{*}=e$, т. е. подгруппа блочных матриц вида

$$
k=\left(\begin{array}{ll}
\alpha & \beta \\
\beta & \alpha
\end{array}\right),
$$

где $\alpha \alpha^{*}+\beta \beta^{*}=e_{p}$ и $\alpha \beta^{*}+\beta \alpha^{*}=0$ (разложение Ивасавы).

Пусть $T$ - особое представление подгруппы $P$ в гильбертовом пространстве $H=L_{\nu}^{2}(S)$, определенное в теореме 2 , и $b-$ его нетривиальный коцикл, заданный формулой (4). Обозначим через $H_{0}$ линейное инвариантное подпространство в $H$, натянутое на векторы коцикла $b$.

Лемма 3. (1) Подпространство $H_{0}$ всюду плотно в $\mathrm{H}$.

(2) Векторы $b(p), p \neq e$, линейно независимы (свойство невырожденности).

Oпределим действия операторов $T(k), k \in K$, на множестве векторов $b(p)$, $p \in P$, по формуле

$$
T(k) b(p)=b\left(p^{\prime}\right),
$$

где $p^{\prime} \in P$ определяется из соотношения $k p=p^{\prime} k^{\prime}, k^{\prime} \in K$.

3. Операторы $T(k)$ удовлетворяют групповому соотношению $T\left(k_{1} k_{2}\right) b(p)=$ $T\left(k_{1}\right) T\left(k_{2}\right) b(p)$ для любых $k_{1}, k_{2} \in K u p \in P$, а потому порождают представление подгруппы $K$ в подпространстве $H_{0}$.

Теорема 4. Oператорь $T(k), k \in K$, совместно с операторами $T(p), p \in P$, порождают представление всей группъ $U(2,2)$ в подпространстве $H_{0}$, в ко-

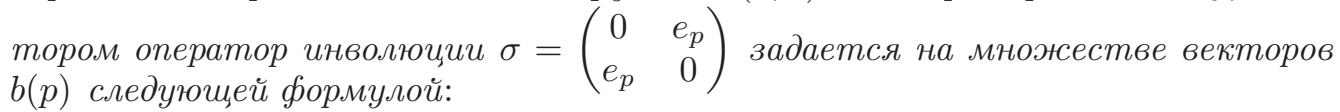

$$
T(\sigma) b(p)=b(\hat{p}), \quad \hat{p} \in P,
$$

где $\hat{p}$ однозначно определяется соотношением $\hat{p} \hat{p}^{*}=\sigma p p^{*} \sigma$. В этом продолэении операторы подгруппы $P$ унитарны, операторы подгруппы $K$ ограничены, $a$ оператор инволючии неограничен и не продолэсается на все пространство $H$. Продолюение 1-коиикла b с группъ $P$ на группу $U(2,2)$ задается формулой

$$
b(p q)=b(p) \quad \text { для любъх } p \in P \text { u } q \in Q .
$$

Приложения описанной конструкции к представлениям группы токов будут изложены в другом месте. Ограничение группой $U(2,2)$ в этой работе обусловлено лишь методическими целями: дать простой пример общей теории, которая, как полагают авторы, распространяется на широкий класс полупростых групп Ли и их группы токов.

\section{ЛитеРАТУРА}

[1] А. М. Вершик, С. И. Карпушев, Когомологии групп в унитарных представлениях, окрестность единищы и условно положительно определенные фучкиии, Матем. сб., 119:4 (1982), 521-533.

[2] Y. Shalom, Rigidity of commensurators and irreducible lattices, Invent. Math., 141:1 (2000), 1-54.

[3] B. Bekka, P. de la Harpe, A. Valette, Kazdan's Property (T), Cambridge University Press, Cambridge, 2007.

[4] U. Haagrup, An example of nonnuclear $C^{*}$-algebra, which has the metric approximation property, Invent. Math., 50:3 (1978), 279-293.

[5] P-A. Cherix, et al., Groups with the Haagerup Property: Gromov's A-T-Menability, Birkhäuser Verlag, Basel, 2001. 
[6] B. Kostant, On existence and irreducibility of certain series of representations, Bull. Amer. Math Soc., 75 (1969), 627-642.

[7] А. М. Вершик, И. М. Гельфанд, М. И. Граев, Представления группь $S L(2, R)$, где $R$ - кольио функиий, УМН, 28:5(173) (1973), 83-128.

[8] А. М. Вершик, И. М. Гельфанд, М. И. Граев, Неприводимые представления груп$n$ и $G^{X}$ и когомологии, Функц. анализ и его прил., 8:2 (1974), 67-69.

[9] I. M. Gelfand, M. I. Graev, A. M. Vershik, Models of representations of current groups, in: Representations of Lie groups and Lie algebras (Budapest, 1971), Akad. Kiadó, Budapest, 1985, 121-179.

[10] R. S. Ismagilov, Representations of infinite-dimensional groups, Transl. Math. Monographs, vol. 152, Amer. Math. Soc., Providence, RI, 1996.

[11] A. Guichardet, Sur la cohomologie des groupe topologiques II, Bull. Soc. Math. France, 96 (1972), 305-332.

[12] P. Delorm, 1-cohomologie des représentations unitaires des groupes de Lie semisimples et résolubes. Produits tenoriels continus et représentations, Bull. Soc. Math. France, 105 (1977), 281-336.

[13] Ф. А. Березин, Представления непрерывного прямого произведения универсальных накрывающих группъ движений комплексного шара, Труды ММО, 36 (1978), 275-293.

[14] H. Araki, Factorisable representations of current algebra, Publ. Res. Inst. Math. Sci., Kyoto Univ., Ser. A, 5 (1969/1970), 361-422.

[15] K. Parthasarathi, K. Schmidt, Positive Definite Kernels, Continuous Tensor Products, and Central Limit Theorems of Probability Theory, Springer-Verlag, Berlin-New York, 1972.

[16] A. M. Vershik, M. I. Graev, The basic representation of the current group $O(n, 1)^{X}$ in the $L^{2}$ space over the generalized Lebesgue measure, Indag. Math., 16:3/4 (2005), 499-529.

[17] А. М. Вершик, М. И. Граев, Структура дополнителънъх серий и особъх представлений групn $S O(n, 1)$ и $S U(n, 1)$, УМH, 61:5 (2006), 3-88.

[18] А. М. Вершик, М. И. Граев, Интегральные модели представлений групп токов, Функц. анализ и его прил., 42:1 (2008), 22-32.

[19] А. М. Вершик, М. И. Граев, Интегральные модели представлений групп токов npocmыx zpynn Лu, УМH, 64:2(386) (2009), 5-72.

[20] А. М. Вершик, М. И. Граев, Пуассонова модель фоковского пространства и представления групп токов, Алгебра и анализ, 23:3 (2011), 63-136.

[21] А. М. Вершик, М. И. Граев, Особые представления групn $U(n, 1)$ u $O(n, 1) u$ связанные с ними представления групп токов $U(\infty, 1)$ и $O(\infty, 1)$ в квазипуассоновом пространстве, Функц. анализ и его прил., 46:1 (2012), 1-12.

[22] А. М. Вершик, Существует ли мера Лебега в бесконечномерном пространстве?, Труды МИАН, 259 (2007), 256-281.

[23] A. M. Vershik, Invariant measures for the continual Cartan subgroup, J. Funct. Anal., 255:9 (2008), 2661-2682.

[24] A. M. Vershik, The behavior of the Laplace transform of the invariant measure on the hypersphere of high dimension, J. Fixed Point Theory Appl., 3:2 (2008), 317-329.

[25] Э. Б. Винберг, А. Л. Онищик, Семинар по группам Ли и алгебраическим группам, Наука, M, 1988.

Санкт-Петербургское отделение Математического института им. В. А. Стеклова РАН

Поступило в редакцию Санкт-Петербургский государственный университет 22 января 2014 г.

e-mail: avershik@gmail.com

e-mail: graev_36@mtu-net.ru 